\title{
Sensibilidad, especificidad y fiabilidad de la escala POP en relación con APACHE II como predictores de pancreatitis aguda grave de origen biliar
}

\author{
Sensitivity, specificity and reliability of the POP score vs. APACHE II score as predictors \\ of severe acute biliary pancreatitis
}

\author{
Rubén Escobar-Arellano', Edmundo Guraieb-Barragán ${ }^{1}$, Antonia Mansanares-Hernández ${ }^{2}$ y \\ Enrique A. Sánchez-Valdivieso $0^{1,3 *}$ \\ ${ }^{1}$ Departamento de Cirugía, Hospital de Alta Especialidad de Veracruz, Secretaría de Salud; '2Departamento de Investigación, Hospital de Alta \\ Especialidad de Veracruz, Secretaría de Salud; ${ }^{3}$ Departamento de Investigación, Escuela de Medicina, Universidad Cristóbal Colón, Campus \\ Calasanz, Boca del Río. Veracruz, México
}

\begin{abstract}
Resumen
Antecedentes: La escala APACHE II predice la gravedad de la pancreatitis aguda, si bien requiere la recolección de un gran número de parámetros. La escala POP, propuesta para estratificar el riesgo de gravedad, requiere pocos parámetros y las variables se recolectan en las primeras 24 horas. Objetivo: Comparar las escalas POP y APACHE II como predictores de gravedad de la pancreatitis al ingreso. Método: Estudio analítico, observacional, en adultos de ambos sexos con pancreatitis aguda de origen biliar. Se aplicaron las escalas APACHE II y POP en las primeras 24 horas del ingreso. Se analizaron el ingreso en la unidad de cuidados intensivos (UCl) y la mortalidad. Resultados: Se incluyeron 208 pacientes (110 mujeres y 98 varones), con una edad de $42.8 \pm 16.6$ años, 59 (28.4\%) de ellos clasificados como pancreatitis grave, e ingresaron en la UCI 20 (9.6\%). La escala POP mostró un $64.1 \%$ de sensibilidad y un $88.2 \%$ de especificidad, mientras que para APACHE II la sensibilidad fue del $66.7 \%$ y la especificidad fue del $87.6 \%$. Conclusiones: La escala POP es una herramienta fiable y fácil de aplicar para estratificar el riesgo de gravedad en pacientes con pancreatitis aguda, con el fin de proporcionar un manejo oportuno y disminuir las complicaciones y la tasa de mortalidad.
\end{abstract}

PALABRAS CLAVE: APACHE II. Escala POP. Gravedad. Pancreatitis aguda.

\begin{abstract}
Background: The APACHE II score predicts severity of acute pancreatitis, although it requires the collection of a large number of parameters. The paediatric observation priority $(P O P)$ score proposed to stratify the risk of severity requires few parameters and the variables are collected in the first $24 \mathrm{~h}$. Objective: To compare POP and APACHE II scores as predictors of the severity of pancreatitis on admission. Methods: Analytical, observational study in adults of both sexes with acute biliary pancreatitis; APACHE II and POP scores were administered in the first $24 \mathrm{~h}$ of admission; We analyzed admission to the intensive care unit (ICU) and mortality. Results: 208 patients were included, 110 women and 98 men, age $42.8 \pm 16.6$ years, classified as severe pancreatitis 59 (28.4\%) patients, requiring ICU 20 (9.6\%) of them. The POP score showed 64.1\% sensitivity and $88.2 \%$ specificity, while for APACHE II score the sensitivity was $66.7 \%$ and the specificity $87.6 \%$. Conclusions: The POP score is a reliable and easy to apply tool to stratify the risk of severity in patients with acute pancreatitis to provide timely management and decrease complications and mortality rate.
\end{abstract}

KEY WORDS: Acute pancreatitis. APACHE II. POP score. Severity.

\section{Correspondencia:}

*Enrique A. Sánchez-Valdivieso

Av. 20 de Noviembre, 1074, Centro

Fecha de recepción: 14-08-2018

C.P. 91700 , Veracruz, Ver., México

Fecha de aceptación: 10-10-2018

E-mail: easanchezv@gmail.com

DOI:10.24875/CIRU.18000662

Cir Cir. 2019;87:402-409

Contents available at PubMed www.cirugiaycirujanos.com 


\section{Introducción}

La pancreatitis aguda (PA) es una de las enfermedades gastrointestinales agudas más importantes en gran parte del mundo desarrollado. La incidencia agrupada bruta de PA en un metanálisis fue de 337 casos por millón de personas-año, pero la incidencia informada en los estudios individuales varía de 150 a 837 casos por millón'. Con frecuencia los pacientes sufren complicaciones, como insuficiencia respiratoria, sepsis, choque, falla renal, etc., que resultan en mayor morbimortalidad $^{2}$. Predecir la pancreatitis aguda grave (PAG) es un objetivo deseable, por lo que continúa la búsqueda del predictor de gravedad ideal. Después de décadas de estratificar la gravedad según la morfología, ahora se reconoce el papel de los aspectos sistémicos en términos de gravedad y persistencia de la falla orgánica relacionada con la pancreatitis.

Se define como $\mathrm{PAG}^{3}$ aquella con presencia de falla orgánica persistente; hasta el $20 \%$ de los pacientes desarrollarán PAG, con una mortalidad que oscila entre el 29 y el $43 \% 4$.

La escala de Ranson, que ha sido usada por mucho tiempo para evaluar la gravedad de la PA, es moderadamente exacta en la clasificación de los pacientes y requiere 48 horas para que sea completada ${ }^{5}$, por lo que se retrasa el manejo oportuno6-8.

La escala APACHE II (Acute Physiology and Chronic Health Evaluation II) fue diseñada originalmente como predictor de supervivencia en la unidad de cuidados intensivos (UCl); es útil para evaluar variables fisiológicas en las primeras 24 y 48 horas. En 1990 se validó su utilización como índice pronóstico en pancreatitis ${ }^{9}$, y una puntuación mayor de 8 predice en un $68 \%$ ataques graves de $\mathrm{PA}^{6-8}$, si bien requiere la recolección de un gran número de parámetros, algunos no relevantes para el pronóstico de la PA, mientras que otros son pasados por alto, como el daño pancreático, las complicaciones regionales, la edad, etc.

Harrison, et al. $^{4}$ presentaron la escala POP (Pancreatitis Outcome Prediction) para estratificar a pacientes con riesgo de PA grave. Sus múltiples ventajas son que se requieren pocos parámetros, que las variables se recolectan en las primeras 24 horas y que su sensibilidad se reporta similar a la de la escala APACHE II.

El objetivo de este estudio fue comparar las escalas POP y APACHE II en PA de origen biliar (PAB) en población mexicana, como predictores de gravedad al ingreso.

\section{Método}

Se realizó un estudio analítico, observacional, en pacientes de ambos sexos, de 18 años de edad o más, que acudieron al servicio de urgencias del Hospital de Alta Especialidad de Veracruz con sospecha diagnóstica de $P A B$, en las primeras 24 horas del ingreso. El estudio fue aprobado por el comité institucional de ética.

\section{Criterios de inclusión}

Se incluyeron pacientes con diagnóstico de PA en el servicio de urgencias; pacientes adultos, de ambos sexos; que cumplan con el criterio propuesto para considerarse como PAG; que tengan una historia clínica con datos completos; formato de consentimiento informado firmado; pacientes con colecistitis crónica calculosa asociada con PA, demostrada por valores séricos elevados de amilasa y lipasa, y hallazgos ecográficos de pancreatitis.

\section{Criterios de exclusión}

Se excluyeron pacientes con infección por el virus de la inmunodeficiencia humana, embarazadas y pacientes referidos de otras unidades con el diagnóstico y el tratamiento establecidos; aquellos con información incompleta; pacientes con enfermedad respiratoria, cardiaca, hematológica, renal, neurológica o hepática crónica previa al ingreso; pacientes con procesos agudos infecciosos previos o concomitantes, no relacionados con la pancreatitis; pseudoquiste pancreático, absceso pancreático, fístula pancreática.

\section{Criterios de eliminación}

Fueron eliminados del estudio los pacientes con egreso antes de las 48 horas o con alta voluntaria.

\section{Definiciones}

La PA se definió como una enfermedad inflamatoria del páncreas de inicio reciente en la que no es posible evidenciar cambios anatómicos que reflejen cronicidad.

La PAB se definió como dolor en el cuadrante superior derecho, un aumento de tres veces en los valores de amilasa sérica y la presencia de cálculos en la vesícula biliar o en los conductos biliares. 
Tabla 1. Parámetros investigados en la escala POP

\begin{tabular}{|c|c|c|c|c|c|c|c|c|c|c|}
\hline \multicolumn{11}{|c|}{ Puntaje en la escala POP } \\
\hline Variable & 0 & 1 & 2 & 3 & 4 & 5 & 6 & 7 & 8 & 10 \\
\hline Edad (años) & $16-29$ & $30-39$ & & $40-49$ & & $50-59$ & & $60-69$ & $>70$ & \\
\hline Presión arterial media (mmHg) & $>90$ & $80-89$ & & $60-79$ & $50-59$ & & $40-49$ & & $<40$ & \\
\hline $\mathrm{PaO}_{2} / \mathrm{FiO}_{2}$ & $>225$ & & & $75-224$ & $<75$ & & & & & \\
\hline $\mathrm{pH}$ arterial & $>7.35$ & $7.30-7.35$ & $7.25-7.24$ & & $7.20-7.24$ & 7.10-7.19 & $7.00-7.09$ & & & $<7$ \\
\hline Urea (mg/dl) & $<14$ & $14-22.3$ & & $22.4-30.7$ & $30.8-47.5$ & & $>47.6$ & & & \\
\hline Calcio (mg/dl) & $8-9.19$ & $7.2-7.99$ & 6.4-7.19; 9.2-9.99 & & $6.40 ;>10$ & & & & & \\
\hline
\end{tabular}

Fuente: Harrison et al. ${ }^{4}$

La PAG se definió ${ }^{3}$, para fines de esta investigación, como aquella con presencia de falla orgánica persistente (o necrosis peripancreática infectada). La definición de gravedad de la PA se basa en los atributos del determinante local (necrosis peripancreática infectada) y el determinante sistémico (falla orgánica persistente) ${ }^{3}$. El determinante sistémico de la gravedad es un grado de disfunción de órganos distantes debido a la PA, cubierto por el término «falla orgánica». La falla orgánica es la principal determinante de la mortalidad. Si la infección está también presente, el impacto es mucho mayor ${ }^{10}$.

La falla orgánica se definió para tres sistemas de órganos (cardiovascular, renal y respiratorio) sobre la base de la peor medición en un periodo de 24 horas. En pacientes sin disfunción orgánica preexistente, la insuficiencia orgánica se define como una puntuación de 2 o más en la escala SOFA (Sepsis related Organ Failure Assessment) o cuando se incumple el umbral pertinente:

- Cardiovascular: necesidad de un agente inotrópico.

- Renal: creatinina $\geq 171 \mu \mathrm{mol} / \mathrm{l} o \geq 2.0 \mathrm{mg} / \mathrm{dl}$.

- Respiratorio: $\mathrm{PaO}_{2} / \mathrm{FiO}_{2}$ (presión parcial de oxígeno en sangre arterial frente a fracción inspirada de oxígeno) $\leq 300 \mathrm{mmHg}$ o $40 \mathrm{kPa}$.

La falla orgánica persistente la definimos como datos de falla orgánica que no se resuelven en un periodo corto de tiempo tras aplicar las medidas de soporte adecuadas.

Fueron clasificados como pacientes con respuesta inflamatoria sistémica (RIS) los que presentaban dos o más de los siguientes datos: frecuencia cardíaca $>90$ latidos/min, frecuencia respiratoria > 20 respiraciones/min o presión parcial de dióxido de carbono $<32 \mathrm{mmHg}$, temperatura corporal $<360>38^{\circ} \mathrm{C}$, conteo de leucocitos $<4000$ o $>12,000 / /$, o $>10 \%$ de neutrófilos inmaduros (bandas).

\section{Instrumentos}

Se aplicaron las dos diferentes escalas, POP y APACHE II, a cada uno de los pacientes al ingreso hospitalario. La escala POP (publicada en 2007$)^{4}$ es una tabla de valoración (Tabla 1) cuya suma da un máximo de 40 puntos e informa de la probabilidad de que el paciente tenga un cuadro de PAG ${ }^{4}$. La escala APACHE II (publicada en 1985) se validó como índice pronóstico en pancreatitis en 1990, con altas sensibilidad y especificidad ${ }^{11}$.

Independientemente de recabar las escalas POP y APACHE II en cada paciente, el cirujano a cargo solicitó estudios complementarios de acuerdo con el caso. En la totalidad de los pacientes se solicitaron hemograma, bioquímica sanguínea completa y análisis de orina. Al mejorar, se continuó el seguimiento como paciente ambulatorio al menos por 30 días.

Se obtuvieron las puntuaciones totales y se clasificaron los pacientes con diagnóstico de PAG según la escala APACHE II, y se compararon con la clasificación de PAG de la escala POP, observando además a los pacientes que ingresaron en la $\mathrm{UCl}$ y la mortalidad asociada a la pancreatitis.

\section{Análisis estadístico}

- Estadística descriptiva: se determinaron las medidas de tendencia central (las medias aritméticas) y las medidas de dispersión (las desviaciones estándar) de las diferentes variables del estudio.

- Estadística inferencial: se utilizó la prueba t de Student para evaluar la diferencia de medias 
entre los grupos; para datos cualitativos o categóricos se evaluaron las diferencias entre los grupos mediante la prueba de ji al cuadrado.

Se realizó el análisis estadístico para determinar la sensibilidad y la especificidad de la escala POP según el puntaje obtenido, y fue comparada con la escala APACHE II al ingreso hospitalario. Se realizó estadística descriptiva de los datos demográficos de la población, así como análisis para pruebas diagnósticas (sensibilidad, especificidad, valor predictivo positivo [VPP], valor predictivo negativo [VPN]); se determinó el punto de corte para las mayores sensibilidad y especificidad de la prueba. Con estos datos se generaron las curvas ROC (Receiver Operating Characteristic) para contrastar ambas escalas, utilizando el programa estadístico SPSS v24.0. Posteriormente se calculó la razón de verosimilitud.

Se compararon los resultados de sensibilidad de ambas clasificaciones entre pacientes con PAG. Del mismo modo, entre los pacientes sin PAG se compararon los resultados en términos de especificidad (prueba de McNemar para datos emparejados). Así mismo, se compararon los valores del área bajo la curva $(A B C)$ de ambas ROC (técnica DeLong, software Med Cal).

En el manejo de los datos se mantuvo la confidencialidad y se respetó el anonimato de los pacientes; uno de los investigadores se hizo cargo de codificar los datos, anotando números en lugar de nombres.

\section{Resultados}

Se incluyeron pacientes clasificados como PAB y $P A B$ grave. Fueron incluidos en este estudio un total de 208 pacientes (46\%); de ellos, 110 (53.2\%) eran mujeres y 98 (47.8\%) eran hombres (Tabla 2). La edad media fue de $42.8 \pm 16.6$ años (rango: 18-90 años). Por lo tanto, la distribución de los casos fue predominante en el sexo femenino y entre la cuarta y la quinta décadas de la vida. Dentro de la comorbilidad, $50(24 \%)$ pacientes presentaban diabetes mellitus tipo 2 y $32(15.4 \%)$ pacientes presentaban hipertensión arterial. Fueron clasificados como PAG 59 (28.4\%) pacientes. Todos los pacientes con PAG mostraron datos de RIS. Ingresaron en la UCI 20 (9.6\%) pacientes, y fallecieron dos de ellos.

Los menores de 40 años (1-2 puntos en la escala POP, 53 [25.5\%] hombres y 61 [29.3\%] mujeres) no pasaron a la UCI. Los pacientes del grupo de 40-59 años (3-5 puntos en la escala POP, 36 [17.3\%] hombres y $34[16.3 \%]$ mujeres) de forma global sumaron
Tabla 2. Distribución de las variables de los pacientes incluidos $(n=208)$

\begin{tabular}{|c|c|c|c|}
\hline & N & $\%$ & Promedio \\
\hline \multicolumn{4}{|l|}{ Sexo } \\
\hline Femenino & 110 & 53.2 & \\
\hline Masculino & 98 & 47.8 & \\
\hline \multicolumn{4}{|l|}{ Edad } \\
\hline Rango 18-90 años & 208 & 100.0 & $42.8 \pm 16.6$ años \\
\hline \multicolumn{4}{|l|}{ Comorbilidad* $^{*}$} \\
\hline Diabetes mellitus tipo 2 & 50 & 24.0 & \\
\hline Hipertensión arterial & 32 & 15.4 & \\
\hline $\begin{array}{l}\text { Pancreatitis aguda grave (con/sin } \\
\text { respuesta inflamatoria sistémica) }\end{array}$ & 59 & 28.4 & \\
\hline Ingreso en $\mathrm{UCl}$ & 20 & 09.6 & \\
\hline Días de estancia en $\mathrm{UCl}$, rango 2-8 días & & & 5.2 \\
\hline \multicolumn{4}{|l|}{ Resultado } \\
\hline Mortalidad en UCI & 2 & 10.0 & \\
\hline Mortalidad hospitalaria & 2 & 01.0 & \\
\hline
\end{tabular}

*Más de un paciente presentó más de una enfermedad concomitante. UCl: unidad de cuidados intensivos.

4 días de estancia (media: 0.1 días) en la UCl. Los días de estancia en la UCI para los 24 (11.5\%) pacientes del grupo de mayores de 60 años (7-8 puntos en la escala POP, 9 [4.3\%] hombres y 15 [7.2\%] mujeres) sumaron 28 (media: 3.0 días), siendo la diferencia estadísticamente significativa en comparación con los menores de 60 años (t de Student, $p=0.0001$ ).

Un factor que resultó bastante llamativo fue el resultado de las cifras de leucocitos en sangre periféri$\mathrm{ca}<4000 \mathrm{o}>12,000$ (que junto con alteraciones en los signos vitales es indicativo de RIS), ya que este resultado se encontró en el $55.6 \%$ de los varones mayores de 60 años con PA, mientras que solo se observó en el $40 \%$ de las mujeres mayores de 60 años; entre los pacientes menores de 60 años se encontró con más frecuencia en las mujeres (21.1\%) que en los varones (12.3\%). Esto sugiere que el factor leucocitos es más relevante en los varones mayores de 60 años, mientras que pierde cierta relevancia en los varones menores de esta edad.

Al aplicarlas al ingreso hospitalario, la escala POP demostró similar certeza diagnóstica que la escala APACHE II (Tabla 3). El punto de corte para las mayores sensibilidad y especificidad de APACHE II fue 8. Para la escala POP, el punto de corte fue 10 , con una sensibilidad del $64.1 \%$ y una especificidad del $88.2 \%$, un VPP de 0.59 y un VPN de 0.95 (con punto de corte en 8 para POP, la sensibilidad fue del $79.5 \%$ y la especificidad fue del $65.3 \%$ ), mientras que para 
Tabla 3. Tabla de contingencia donde los resultados fueron obtenidos

\begin{tabular}{lccc}
\hline \multicolumn{4}{c}{ Escala POP } \\
\hline Gravedad & $\begin{array}{c}\text { Pancreatitis aguda } \\
\text { grave positivo }\end{array}$ & $\begin{array}{c}\text { Pancreatitis aguda } \\
\text { no grave negativo }\end{array}$ & Total \\
\hline Negativo & 9 & 162 & 171 \\
Positivo & 22 & 15 & 37 \\
Total & 31 & 177 & 208 \\
& FN 9 & VN 162 & \\
& VP 22 & FP 15 & \\
\hline
\end{tabular}

\begin{tabular}{lccc}
\hline \multicolumn{4}{c}{ Escala APACHE II } \\
\hline Gravedad & $\begin{array}{c}\text { Pancreatitis aguda } \\
\text { grave positivo }\end{array}$ & $\begin{array}{c}\text { Pancreatitis aguda } \\
\text { no grave negativo }\end{array}$ & Total \\
\hline Negativo & 7 & 144 & 151 \\
Positivo & 24 & 33 & 57 \\
Total & 31 & 177 & 208 \\
& FN 7 & VN 144 & \\
& VP 24 & FP 33 & \\
\hline
\end{tabular}

\begin{tabular}{lccc}
\hline \multicolumn{4}{c}{ Escala POP } \\
\hline Sensibilidad & Especificidad & VPP & VPN \\
\hline 0.64 & 0.88 & 0.59 & 0.95 \\
\hline
\end{tabular}

\begin{tabular}{lccc}
\hline \multicolumn{4}{c}{ Escala APACHE II } \\
\hline Sensibilidad & Especificidad & VPP & VPN \\
\hline 0.66 & 0.87 & 0.42 & 0.95 \\
\hline
\end{tabular}

FN: falso negativo; FP: falso positivo; VN: verdadero negativo; VP: verdadero positivo; VPN: valor predictivo negativo; VPP: valor predictivo positivo.

la determinación de APACHE II al ingreso se obtuvo una sensibilidad del $66.7 \%$, una especificidad del $87.6 \%$, un VPP de 0.42 y un VPN de 0.95 (Tabla 3).

La escala POP muestra un ABC de 0.817 (intervalo de confianza del 95\% [IC 95\%]: 0.733-0.901), que fue superior a APACHE II (ABC de 0.783; IC 95\%: 0.6810.885) (Fig. 1).

La razón de verosimilitud $(\mathrm{RV})+$ (cociente entre la sensibilidad y 1 - especificidad) de la escala POP fue 5.33, mientras que para APACHE II fue 5.08. La RV(cociente entre 1 - sensibilidad y la especificidad) de POP fue 0.41, y la de APACHE II fue 0.39. La probabilidad de que un individuo fuera clasificado correctamente (valor global) en ambas pruebas fue de 0.8846 para POP y de 0.8077 para APACHE II.

Se compararon los resultados de sensibilidad entre pacientes con PAG y, del mismo modo, los resultados de especificidad entre los pacientes sin PAG. Las diferencias observadas entre las puntuaciones de

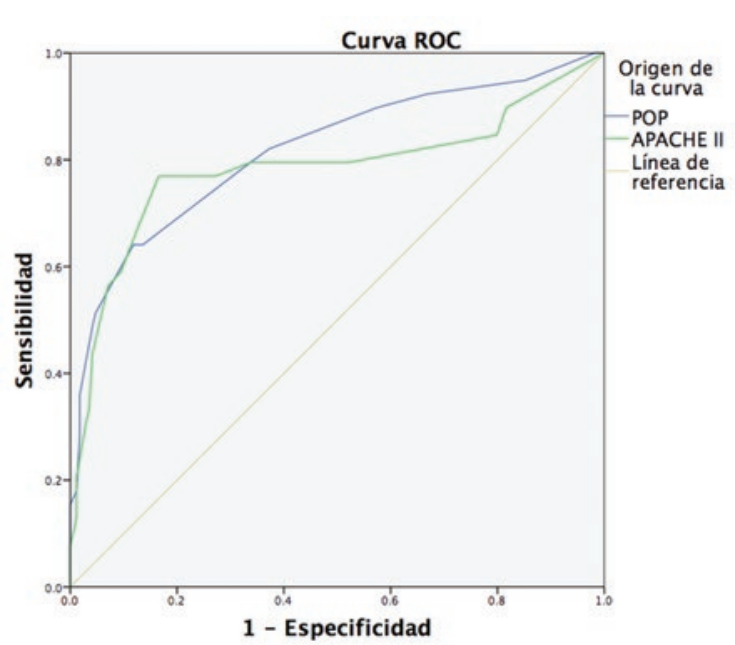

Figura 1. Curva ROC obtenida calculando los resultados de ambas escalas. Escala POP: área bajo la curva $=0.817$ (IC 95\%: 0.7330.901). Escala APACHE II: área bajo la curva $=0.783$ (IC 95\%: 0.681 0.885). Los segmentos de diagonal se generan mediante empates.

ambas escalas no fueron estadísticamente significativas (prueba de McNemar para datos emparejados), así como tampoco lo fue la diferencia entre los valores de $A B C$ de ambas curvas ROC (técnica DeLong, software Med Cal).

Por medio del análisis de intersección (teorema de Bayes) se encontró que, para la escala POP, el $82.2 \%$ de la muestra fue por POP negativo para gravedad; el restante $17.8 \%$ fue según POP positivo para gravedad. Solo el $5.3 \%$ de la muestra fue PAG+, aunque POP fue negativo para gravedad y el $94.7 \%$ fue PAG- determinado por POP (negativo para gravedad). El $59.5 \%$ de la muestra es $P A G+$, dado que POP fue positivo para gravedad; el $40.5 \%$ de la muestra es PAG-, aunque POP es positivo para gravedad. Por el contrario, para la escala APACHE II el $72.6 \%$ de la muestra es APACHE II negativo para gravedad; el restante $27.4 \%$ de la muestra es APACHE II positivo para gravedad. Solo el $4.6 \%$ fue PAG+, aunque APACHE II fue negativo para gravedad; el $95.4 \%$ fue PAG- dado que APACHE II fue negativo para gravedad. El $42.1 \%$ fue $P A G+$ dado que APACHE II es positivo para gravedad, si bien el 57.9\% fue PAG-, aunque APACHE II es positivo para gravedad.

\section{Discusión}

La proporción esperada de casos graves es cercana al $12-15 \%{ }^{12,13}$. Es muy conveniente anticipar la gravedad de cada caso en particular, lo que mejorará los resultados y disminuirá los costos. El objetivo de este 
estudio fue valorar la utilidad de la escala POP para la estratificación de la PAB como grave o no grave, en población mexicana, tomando como parámetro la puntuación APACHE II validada como índice pronóstico en pancreatitis ${ }^{11}$, que tiene la ventaja de poder realizarse en las primeras 24 horas, pero tiene el gran inconveniente de que requiere muchas variables y un gran consumo de tiempo y de recursos.

En 2007, Harrison, et al. ${ }^{4}$ describieron la escala POP, que se deriva de los datos de la cohorte ICNARC (Intensive Care National Audit \& Research Centre, del Reino Unido) y se basa en el pH arterial, la edad, la urea sérica, la presión arterial media, la relación $\mathrm{pO}_{2} / \mathrm{FiO}_{2}$ y el calcio sérico total (en orden decreciente de impacto). Aunque en el artículo original mostró superioridad sobre las otras escalas, aún se está validando en diferentes cohortes de pacientes ${ }^{14}$.

Un estudio que incluyó pacientes diagnosticados de $\mathrm{PA}$ en la $\mathrm{UCl}^{15}$ encontró que la escala POP tenía un poder discriminativo claramente superior en comparación con otros índices de gravedad relacionados con la mortalidad de los pacientes en la $\mathrm{UCl}$ y en el hospital. Los autores reportan un $A B C$ de la curva ROC de 0.899 para la escala POP a 0 horas en UCI y de 0.812 a 0 horas en hospitalización, entre 108 pacientes, el $50 \%$ de ellos mujeres ${ }^{15}$, comparándola favorablemente con otros índices de gravedad, aunque la edad media de sus pacientes fue de $58.7 \pm$ 15.8 años (rango: 18-88 años), mucho mayor que la nuestra (42.8 \pm 16.6 ; rango: $18-90$ años), y la etiología fue más variada.

En nuestro análisis se encontró una sensibilidad del $64.1 \%$ y una especificidad de $88.2 \%$ para la escala POP en la estimación de la gravedad de la PA, que resultó ligeramente superior a la más frecuentemente utilizada escala APACHE II. Para Mounzer, et al. ${ }^{16}$, APACHE II y POP son las mejores escalas para predecir falla orgánica en pacientes con PA.

Papachristou, et al. ${ }^{5}$ compararon las escalas POP, APACHE II y Ranson investigadas al ingreso del paciente en relación con las complicaciones y la gravedad de la pancreatitis, y observaron una estrecha relación entre la gravedad de la PA y el puntaje de la escala POP $>8$.

En nuestro estudio, la $R V+$ fue de 5.33 para la escala POP y de 5.08 para la escala APACHE II. Comparativamente, se calculó la $\mathrm{RV}+$ para el estudio de Mounzer, et al..$^{16} \mathrm{y}$ resultó de 2.38 para la escala POP y de 2.90 para la escala APACHE II (sensibilidad del $57 \%$ y especificidad del $76 \%$ para POP, y sensibilidad del $84 \%$ y especificidad del $71 \%$ para APACHE II). No son tampoco muy diferentes las $\mathrm{RV}+$ de ambas escalas.

Pavlidis, et al. ${ }^{17}$, en su estudio en la UCI, compararon las escalas clásicas APACHE II y SOFA con otras como las escalas POP y Balthazar. Obtuvieron unos puntajes medios al ingreso en la UCI para APACHE II de 7 puntos, para POP de 8 puntos, para SOFA de 5 puntos y para Balthazar de 4 puntos, sin diferencia significativa entre las escalas APACHE II y POP, ambas con unas altas sensibilidad y especificidad, al igual que en nuestro estudio. En cuanto a la evaluación del riesgo, no hubo diferencias significativas en los puntajes de APACHE II y de otras escalas entre los sobrevivientes del hospital y los que no sobrevivieron. En cambio, los pacientes que murieron en el hospital tenían una puntuación de POP significativamente mayor al ingreso (13; rango: 11-19) en comparación con los pacientes sobrevivientes del hospital (7; rango: $4.5-11)^{17}$. La escala POP tuvo un mayor $A B C$ de la curva ROC, pero la diferencia entre POP y APACHE no fue estadísticamente significativa (ABC 0.84 vs. 0.68 , respectivamente $)^{17}$. De modo similar, en nuestro estudio la escala POP tuvo un $A B C$ de la curva ROC mayor que la escala APACHE II (ABC 0.82 vs. 0.78 , respectivamente), pero la diferencia no fue estadísticamente significativa. En el estudio de Monzer, et al. ${ }^{16}$, el ABC para APACHE II fue mayor que para $P O P$ ( $A B C 0.77$ vs. 0.67 , respectivamente), si bien los puntos de corte que aplicaron estos últimos autores fueron diferentes (7 para APACHE II y 9 para POP).

En otro estudio sobre riesgo para gravedad de la $\mathrm{PA}^{18}$, los pacientes clasificados con PAG mostraron dos o más indicadores de RIS; similarmente, en nuestro estudio observamos que todos los pacientes clasificados con RIS tuvieron PAG.

Harrison, et al. ${ }^{4}$ reportaron en su estudio de validación de la escala POP con 2462 pacientes con PAG ingresados en la $\mathrm{UCl}$ una sensibilidad del $90 \%$ y una especificidad del $85 \%$, y un $A B C$ de 0.838 , que son superiores a nuestros resultados (sensibilidad del $64.1 \%$, especificidad del $88.2 \%$ y $A B C$ de 0.817 ), pero que son muy similares a aquellos valores obtenidos por APACHE II, escala cuya validez ya ha sido demostrada.

Las ventajas que deseamos destacar de la escala POP son su aplicabilidad y su eficacia. La escala POP es más práctica que la escala APACHE II en este tipo de pacientes, ya que solo toma en cuenta seis variables que se obtienen de forma fácil y al 
ingreso, y realiza una estratificación de la PA permitiendo dirigir el manejo médico dependiente de la gravedad de una manera oportuna, optimizando así los recursos en una forma eficaz y eficiente. Posteriormente es posible valorar a aquellos pacientes que así lo requieran con las escalas tradicionales. Parecería, según nuestros resultados, que APACHE Il tiene una tendencia a ser mejor que POP en cuanto a la predicción de gravedad de la PA en los pacientes menores de 60 años, mientras que lo contrario sucede con la escala POP, que parece ser de mayor utilidad en los pacientes mayores de 60 años.

El factor «cifra de leucocitos < 4000 o > 12,000» es más relevante en los varones mayores de 60 años, mientras que pierde cierta relevancia en los varones menores de 60 años, probablemente por cierta anergia por envejecimiento en especial entre las mujeres. El $30 \%$ de los adultos mayores que tenían dolor abdominal quirúrgico no presentaron fiebre ni leucocito$\mathrm{sis}^{19}$. En los adultos mayores, el 25-32\% no presentó leucocitosis y el $16-28 \%$ carecía tanto de fiebre como de leucocitosis en diferentes patologías quirúrgicas ${ }^{20}$. Por último, en nuestro estudio observamos que todos los pacientes con RIS presentaron PAG, pero no encontramos relación alguna entre el síndrome de RIS y la mortalidad.

Así mismo, observamos que el sexo femenino fue el más frecuentemente afectado en nuestra población, lo que sugiere una asociación entre sexo femenino y PAB; se ha reportado que los pacientes con pancreatitis biliar tienen más probabilidades de ser mayores y mujeres ${ }^{21}$, y que la pancreatitis biliar es más frecuente en las mujeres, lo que al parecer influyó en la tendencia observada en la presente serie ${ }^{13,22}$. Sin embargo, la diferencia es de solo un $6 \%$, por lo que, al no disponer de estadísticas nacionales, no es posible establecer con certeza el comportamiento.

Se propone entonces la utilización sistemática de la escala POP como índice pronóstico, que solo requiere seis parámetros (edad, presión arterial media, índice de Fick, pH arterial, urea sérica y calcio sérico), para estratificar a los pacientes con PAG, ya que es barata, simple y rápida, con una alta especificidad, para así poder proporcionar un manejo oportuno y disminuir las complicaciones y la mortalidad por PA.

\section{Conclusión}

La estratificación de la gravedad no es precisa con respecto a la predicción de la progresión clínica de la
PA. En particular, la identificación temprana de pacientes con alto riesgo de desarrollar las formas más graves y los estudios sobre sistemas pronóstico se han centrado en identificar pacientes con PAG dentro de grupos heterogéneos de pacientes. En este estudio se pudo comprobar la utilidad y la confiabilidad de la escala POP comparada con la escala tradicional APACHE II; demostramos aquí que la escala POP es un excelente predictor de las tasas de mortalidad en una población homogénea con $\mathrm{PAB}$ ( $\mathrm{ABC}$ de la curva $R O C>0.8)$ en comparación con APACHE II.

\section{Conflicto de intereses}

Los autores declaran que no existen conflictos de intereses en relación con esta investigación.

\section{Financiamiento}

Los autores declaran que no se recibió ninguna financiación.

\section{Responsabilidades éticas}

Protección de personas y animales. Los autores declaran que los procedimientos seguidos se conformaron a las normas éticas del comité de experimentación humana responsable y de acuerdo con la Asociación Médica Mundial y la Declaración de Helsinki.

Confidencialidad de los datos. Los autores declaran que han seguido los protocolos de su centro de trabajo sobre la publicación de datos de pacientes.

Derecho a la privacidad y consentimiento informado. Los autores han obtenido el consentimiento informado de los pacientes y/o sujetos referidos en el artículo. Este documento obra en poder del autor de correspondencia.

\section{Bibliografía}

1. Xiao AY, Tan MLY, Wu LM, Asrani VM, Windsor JA, Yadav D, et al. Global incidence and mortality of pancreatic diseases: a systematic review, meta-analysis, and meta-regression of population-based cohort studies. Lancet Gastroenterol Hepatol. 2016;1:45-55.

2. Fagenholz PJ, Fernández-del Castillo C, Harris NS, Pelletier AJ, Camargo CA Jr. Direct medical costs of acute pancreatitis hospitalizations in the United States. Pancreas. 2007;35:302-7.

3. Dellinger EP, Forsmark CE, Layer P, Lévy P, Maraví-Poma E, Petrov MS, et al. Determinant-based classification of acute pancreatitis severity: an international multidisciplinary consultation. Ann Surg. 2012;256:875-80.

4. Harrison DA, D'Amico G, Singer M. The Pancreatitis Outcome Prediction (POP) Score: a new prognostic index for patients with severe acute pancreatitis. Crit Care Med. 2007;35:1703-8.

5. Papachristou GI, Clermont G, Sharma A, Yadav D, Whitcomb DC. Risk and markers of severe acute pancreatitis. Gastroenterol Clin North Am. 2007;36:277-96. 
6. Marshall JC, Cook DJ, Christou NV, Bernard GR, Sprung CL, Sibbald WJ. Multiple organ dysfunction score: a reliable descriptor of complex clinical outcome. Crit Care Med. 1995;23:1638-52.

7. Wall I, Badalov N, Baradarian R, Iswara K, Li JJ, Tenner S. Decreased morbidity and mortality in patients with acute pancreatitis related to aggressive intravenous hydration. Pancreas. 2011;40:547-50.

8. Gardner TB, Vege SS, Pearson RK, Chari ST. Fluid resuscitation in acute pancreatitis. Clin Gastroenterol Hepatol. 2008;6:1070-6.

9. Wilson C, Heath DI, Imrie CW. Prediction of outcome in acute pancreatitis: a comparative study of APACHE II, clinical assessment and multiple factor scoring systems. Br J Surg. 1990;77:1260-4.

10. De Waele JJ. Acute pancreatitis. Curr Opin Crit Care. 2014;20:189-95.

11. Yadav D, Lowenfels AB. Trends in the epidemiology of the first attack of acute pancreatitis: a systemic review. Pancreas. 2006;33:323-30.

12. Anand N, Park JH, Wu BU. Modern management of acute pancreatitis. Gastroenterol Clin North Am. 2012;41:1-8.

13. Reid GP, Williams EW, Francis DK, Lee MG. Acute pancreatitis: a 7 year retrospective cohort study of the epidemiology, aetiology and outcome from a tertiary hospital in Jamaica. Ann Med Surg. 2017;20:103-8.

14. Ledesma-Heyer JP, Arias Amaral J. Pancreatitis aguda. Med Int Mex. 2009;25:285-94.

15. Amálio SM, Macedo MA, Carvalho SM, Moreno RP. Mortality assessment in patients with severe acute pancreatitis: a comparative study of specific and general severity indices. Rev Bras Ter Intensiva. 2012;24:246-51.
16. Mounzer R, Langmead CJ, Wu BU, Evans AC, Bishehsari F, Muddana V, et al. Comparison of existing clinical scoring systems to predict persistent organ failure in patients with acute pancreatitis. Gastroenterology. 2012; 142:1476-82.

17. Pavlidis $P$, Crichton S, Lemmich Smith J, Morrison D, Atkinson S, Wyncoll $D$, et al. Improved outcome of severe acute pancreatitis in the intensive care unit. Crit Care Res Pract. 2013;2013:897107.

18. Mofidi R, Suttie SA, Patil PV, Ogston S, Parks RW. The value of procalcitonin at predicting the severity of acute pancreatitis and development of infected pancreatic necrosis: systematic review. Surgery. 2009; 146:72-81.

19. Potts FE, Vukov LF. Utility of fever and leukocytosis in acute surgical abdomens in octogenarians. J Gerontol A Biol Sci Med Sci. 1999; 54:M55-8.

20. Ragsdale L, Southerland L. Acute abdominal pain in the older adult. Emerg Med Clin North Am. 2011;29:429-48.

21. Nesvaderani M, Eslick GD, Vagg D, Faraj S, Cox MR. Epidemiology, aetiology and outcomes of acute pancreatitis: a retrospective cohort study. Int J Surg. 2015;23:68-74.

22. Almeida N, Fernández A, Casela A. Predictors of severity and in-hospital mortality for acute pancreatitis: is there any role for $\mathrm{C}$-reactive protein determination in the first 24 hours? GE Port J Gastroenterol. 2015; 22:187-9. 